\title{
Pulmonary arterial thrombosis as an important complication of COVID-19 pulmonary disease: letter to the editor
}

\author{
Sigurd F. Lax ${ }^{1,2}$ (D) $\cdot$ Kristijan Skok $^{1}$ (D) Michael Trauner $^{3}$ (D) on behalf of all coauthors \\ Published online: 8 August 2020 \\ (C) Springer-Verlag GmbH Germany, part of Springer Nature 2020
}

To the Editor,

We read with great interest the article "The evolution of pulmonary pathology in fatal COVID-19 disease: an autopsy study with clinical correlation" by Bösmüller and colleagues describing pulmonary changes in 4 patients deceased from COVID-19 [1]. Besides the different stages of diffuse alveolar damage (DAD), the authors also describe vascular changes, in particular micro- and macrothrombosis of pulmonary arteries and infarction or infarct-like changes of the surrounding pulmonary parenchyma in association with endothelitis. It is reassuring to see that this paper convincingly confirms the key findings of our study (published May 14) which has demonstrated pulmonary arterial thrombosis as an important and so far, underrecognized complication of SARS-CoV-2-associated pulmonary disease [2]. In our study on 11 COVID-19 deceased, we also described the different stages of DAD from alveolar edema and hyaline membranes to proliferation of alveolar macrophages and organization by fibrosis. Similar changes have been previously found in SARS-1 but may also be present in acute respiratory distress syndrome (ARDS) by other causes such as the different types of shock. COVID-19 pulmonary arterial thrombosis with subsequent occlusion of small- and mid-sized arteries leads to a massive breakdown of pulmonary circulation and oxygenation. Thrombotic occlusion of pulmonary arteries has been described in the older

This article is part of the Topical Collection on Quality in Pathology

Sigurd F. Lax

sigurd.lax@kages.at

1 Department of Pathology, Hospital Graz II, Academic Teaching Hospital of the Medical University Graz, Graz, Austria

2 School of Medicine, Johannes Kepler University Linz, Linz, Austria

3 Division of Gastroenterology and Hepatology with Intensive Care 13H1, Department of Internal Medicine III, Medical University of Vienna, Vienna, Austria literature as a complication of ARDS including an intent of treatment by streptokinase infusion [3] and more recently in pediatric ARDS. The issue of thromboembolism versus thrombosis in COVID-19 pulmonary disease has led to an intense discussion $[4,5]$. In particular, the location in the subsegmental arteries, the pattern of distribution of the thrombotic material in multiple vessels (as demonstrated in our and the present paper by gross figures), the associated endothelitis, as well as the subtotal or total filling of the vessels favors in situ thrombosis [2]. In addition, these occluded vessels are almost exclusively found in areas of hypostasis, whereas thromboemboli would be randomly distributed and also found in well-ventilated areas of the lungs. Fixation of the total lungs in formalin and serial sectioning as performed by us and others facilitate the detection of these pulmonary arteries occluded by thrombotic material in contrast to a sampling of fresh lung tissue during autopsy [2]. These findings seem to have a tremendous clinical impact, although at the moment, an effective therapeutic strategy is still lacking. Prophylactic anticoagulation is certainly justified but may be ineffective to prevent thrombosis as seen in our study and other studies [2]. On the other hand, therapeutic anticoagulation could be effective but lacks evidence by controlled clinical trials and also has risks such as bleeding into the lung parenchyma and other sites [4].

In conclusion, severe and fatal course of COVID-19 seems to be caused not only by the inflammatory reaction of the lung parenchyma but also frequently by thrombotic occlusion of small- to mid-sized pulmonary arteries and subsequent infarction of lung parenchyma as it can be found in ARDS of different pathogenesis.

Author contributions Drafting of the letter: S.F. Lax; Critical revision: K. Skok, M. Trauner, S.F. Lax

Funding information The authors received no funding with respect to this letter. 


\section{Compliance with ethical standards}

Conflict of interest Dr. Skok declares no conflict of interest. Dr. Lax reports personal fees from Roche, AstraZeneca, Novartis, and Biogena outside the submitted work. Dr. Trauner has received speaker fees from Bristol-Myers Squibb, Falk, Gilead, Intercept and Merck Sharp \& Dohme (MSD); advisory board fees from Albireo, Boehringer Ingelheim, BiomX, Falk, Genfit, Gilead, Intercept, MSD, Novartis, Phenex, and Regulus; travel grants from AbbVie, Falk, Gilead, and Intercept; and research grants from Albireo, CymaBay, Falk, Gilead, Intercept, MSD, and Takeda all outside the submitted work.

\section{References}

1. Bosmuller H, Traxler S, Bitzer M, Haberle H, Raiser W, Nann D, Frauenfeld L, Vogelsberg A, Klingel K, Fend F (2020) The evolution of pulmonary pathology in fatal COVID-19 disease: an autopsy study with clinical correlation. Virchows Arch. https://doi.org/10. 1007/s00428-020-02881-x
2. Lax SF, Skok K, Zechner P, Kessler HH, Kaufmann N, Koelblinger C, Vander K, Bargfrieder U, Trauner M (2020) Pulmonary arterial thrombosis in COVID-19 with fatal outcome: results from a prospective, single-center. Ann Intern Med. https://doi.org/10.7326/M202566

3. Greene R, Lind S, Jantsch H, Wilson R, Lynch K, Jones R, Carvalho A, Reid L, Waltman AC, Zapol W (1987) Pulmonary vascular obstruction in severe ARDS: angiographic alterations after I.V. fibrinolytic therapy. AJR Am J Roentgenol 148(3):501-508. https://doi. org/10.2214/ajr.148.3.501

4. Cattaneo M, Bertinato EM, Birocchi S, Brizio C, Malavolta D, Manzoni M, Muscarella G, Orlandi M (2020) Pulmonary embolism or pulmonary thrombosis in COVID-19? Is the recommendation to use high-dose heparin for thromboprophylaxis justified?. Thromb Haemost. https://doi.org/10.1055/s-0040-1712097

5. Deshpande C (2020) Thromboembolic findings in COVID-19 autopsies: pulmonary thrombosis or embolism?. Ann Intern Med. https://doi.org/10.7326/M20-3255

Publisher's note Springer Nature remains neutral with regard to jurisdictional claims in published maps and institutional affiliations. 